\title{
THE EFFECTS OF MIRTAZAPINE ON SLEEP
}

\author{
A. Jorge ${ }^{1}$, J. Carvalheiro' ${ }^{1}$, L. Paiva Delgado ${ }^{1}$ \\ ${ }^{1}$ Centro Hospitalar do Médio Tejo, Psychiatry, Tomar, Portugal.
}

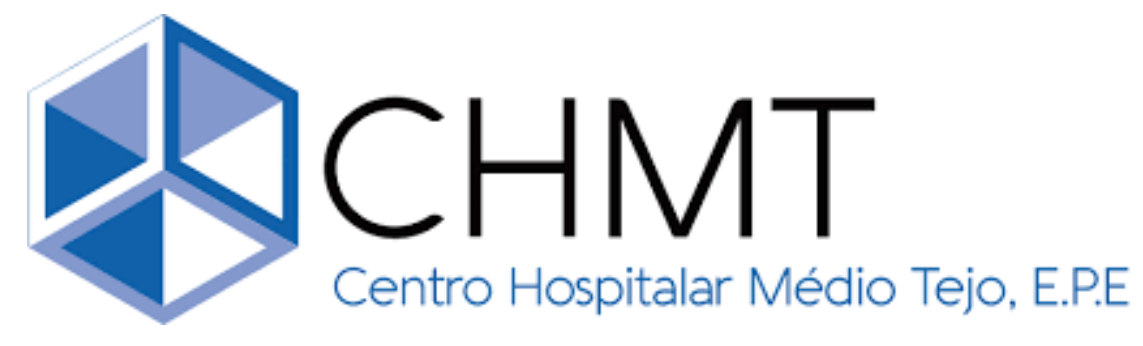

\section{Background and Aims:}

Insomnia, defined as a persistent difficulty with sleep initiation, duration, consolidation and/or quality, that occurs despite adequate opportunity and circumstances for sleep is a highly prevalent condition in the general population.

Mirtazapine is a commonly used antidepressant with a wellknown ability to produce sedation.

Can we use mirtazapine as a hypnotic in our patients?

\section{Materials and Methods:}

Pubmed and medscape was used as bibliographical sources.

\section{Results and Conclusions:}

Insomnia is associated with the development of diverse medical health issues, such as heart diseases. Insomniacs use more medications and have increased health-care utilization compared to healthy individuals. Hypnotics, particularly the benzodiazepines, can have serious disadvantages, such as sleepiness and reduced cognitive performance during the next day, rapid development of tolerance, rebound insomnia upon drug discontinuation, and a serious risk of misuse and abuse.

Mirtazapine was first synthesized and written about in 1989. It was first approved for the treatment of a major depressive disorder in the Netherlands in 1994. Mirtazapine is an atypical antidepressant and is used primarily for the treatment of a major depressive disorder. The drug was also found to have sedative, antiemetic, anxiolytic, and appetite stimulant effects.

A systemic review and network meta-analysis conducted in 2018 comparing the efficacy and acceptability of 21 different antidepressant drugs demonstrated mirtazapine as one of the most effective when compared to other antidepressants in headto-head studies.

In 2011, a Cochrane review found that when compared to other antidepressants, mirtazapine is more likely to cause sleepiness. Mirtazapine also appears to have significantly different effects on electroencephalogram sleep pattern when compared with other antidepressants.

At low doses, like $15 \mathrm{mg} / \mathrm{day}$, the prominent antihistaminergic properties of mirtazapine may result in a nonspecific sedative effect, although this sedative effect seems to be mainly mediated by 5-HT2 receptor blockade.

As with all antidepressants, there are certain side effects associated with Mirtazapine use. These include weight gain and, because the drug remains in one's system throughout the course of the day, a person might also experience drowsiness.

Less common side-effects include producing or exacerbating periodic leg movements in young healthy men which may affect their sleep quality, although this side-effect has only been reported in a recent, small-sample study

In a study on a single dose of mirtazapine given to six human volunteers with normal sleep patterns, mirtazapine significantly shortened the time to onset of sleep, and stage 1 sleep was reduced, while the amount of deep sleep was significantly increased. In addition, mirtazapine significantly increased the latency of rapid eye movement stage 2 sleep and reduced nighttime wakening.
In placebo-controlled studies with depressed patients, mirtazapine has been reported to improve subjective complaints of sleep disturbance.

Mirtazapine produces minimal changes on actigraphic measures of sleep continuity. Research has shown that Mirtazapine, taken at bedtime, to have a positive impact on insomnia symptoms in people with clinical depression. It has also been shown to improve the percentage of time in bed spent asleep, decrease nighttime awakenings and increase total time spent asleep.

Studies have shown a decrease in sleepiness after 2 weeks of mirtazapine administration, and this phenomenon may be attributed to tolerance developed to the antihistamine effects of mirtazapine.

When used in approved doses, i.e. 15-45 mg mirtazapine have been shown to improve sleep efficiency and increase total sleep time in healthy individuals as well as in people with mental disorders.

$7.5 \mathrm{mg}$ of mirtazapine increased sleep considerably compared to placebo, by improving sleep continuity and total sleep time in a model of transient insomnia, with some hangover effects in the morning.

Mirtazapine is an antidepressant with sleep-promoting effects significantly greater than placebo, similar to tricyclic antidepressants, and somewhat similar to selective serotonin reuptake inhibitors and serotonin-norepinephrine reuptake inhibitors. These effects must be balanced with mirtazapine's ability to cause sedation-related side effects.

The beneficial effects of mirtazapine on sleep quality, in addition to its antidepressant activity, are of clinical importance; particularly in members of the elderly population who frequently suffer from sleep complaints and are under simultaneous treatment with several drugs.

Considering all results, mirtazapine might be a suitable option for the treatment of depressed elderly patients with comorbid insomnia and mirtazapine improves sleep quality and decreases the concomitant use of sleep medications.

Sources: 1. Watanabe N, Omori IM, Nakagawa A, Cipriani A, Barbui C, Churchill R, Furukawa TA. Mirtazapine versus other antidepressive agents for depression. Cochrane Database Syst Rev. 2011 Dec 07;(12):CD006528 2. Jilani TN, Saadabadi A. Mirtazapine. [Updated 2019 Mar 24]. In: StatPearls [Internet]. Treasure Island (FL): StatPearls Publishing; 2019 Jan-. Available from: https://www.ncbi.nlm.nih.gov/books/NBK519059/ 3. San, L., \& Arranz, B. (2006). Mirtazapine: only for depression? Acta Neuropsychiatrica, 18(3-4), 130143 4. Rothschild-Fuentes, B., Roche, A., Jiménez-Genchi, A., Sánchez-Ferrer, J., Fresan, A., \& Muñoz-Delgado, J. (2012). 5. Effects of Mirtazapine on the Sleep Wake Rhythm of Geriatric Patients with Major Depression: An Exploratory Study with Actigraphy. Pharmacopsychiatry, 46(02), 59-62 6. Scoralick, F. M., Louzada, L. L., Quintas, J. L., Naves, J. O. S., Camargos, E. F., \& Nóbrega, O. T. (2016). 7. Mirtazapine does not improve sleep disorders in Alzheimer's disease: results from a double-blind, placebo-controlled pilot study. Psychogeriatrics, 17(2), 89-96. 8. Winokur, Andrew \& J Sateia, Michael \& Hayes, J.Boyd \& Bayles-Dazet, Wendy \& MacDonald, Mary \& Gary, Keith. (2000). 9. Acute effects of mirtazapine on sleep continuity and sleep architecture in depressed patients: A pilot study. Biological psychiatry. 48. 75-8. 10.1016/S0006-3223(00)00882-9. 10. Fulda, S., Kloiber, S., Dose, T., Lucae, S., Holsboer, F., Schaaf, L., Hennings, J. (2013). Mirtazapine provokes periodic leg movements during sleep in young healthy men. Sleep, 36(5), 661-669 